\title{
Pattern of physical growth during first six months of life among a cohort of babies living in Gampaha district, Sri Lanka
}

\author{
Priyantha Perera $^{1^{*}}$, Meranthi Fernando ${ }^{1}$, Sachith Meththananda ${ }^{1}$, Rohini Samaranayake $^{2}$ \\ ${ }^{1}$ Department of Paediatrics, Faculty of Medicine, University of Kelaniya, Kelaniya, Sri Lanka; \\ *Corresponding Author: priyanthaprr@gmail.com \\ ${ }^{2}$ Department of Tropical Health, Faculty of Medicine, University of Kelaniya, Kelaniya, Sri Lanka
}

Received 23 June 2013; revised 24 July 2013; accepted 12 August 2013

Copyright (C) 2013 Priyantha Perera et al. This is an open access article distributed under the Creative Commons Attribution License, which permits unrestricted use, distribution, and reproduction in any medium, provided the original work is properly cited.

\begin{abstract}
Introduction: Current WHO recommendation is to continue exclusive breast feeding (EBF) up to six months. Main concern regarding this recommendation is, whether EBF supports rapidly growing infant with adequate nutrition. Methods: A cohort of randomly selected new-borns was followed up at two, four and six months, to study feeding pattern and physical growth. Feeding practices and socio-demographic data were collected using an interviewer administered questionnaire. Weight \& length were measured using standard techniques. Results: EBF rates were very high in this cohort of children. One main reason to stop EBF was growth faltering. Only some children showed a catch up growth by stopping EBF. At two, four and six months, weight faltering rates among EBF babies were, $11.1 \%, 20.1 \%$ and $13.2 \%$ respectively. Length faltering had a similar pattern, but with a higher rate at all ages. Conclusions: EBF up to six months did not cause growth faltering in the majority. In the majority, no identifiable cause for growth faltering was found. Possible genetic influence on growth faltering was not considered when stopping EBF.
\end{abstract}

Keywords: Exclusive Breastfeeding; Length; Growth Faltering; Growth Charts; Growth Monitoring

\section{INTRODUCTION}

Correct feeding practices during early childhood play an important role in physical growth, cognitive development, immunity, prevention of obesity and protection from atopic conditions \& cardio-vascular problems in later life [1]. Breast milk provides adequate nutrients to the baby during early months of life in correct proportions. Since 2001, World Health Organization (WHO) has recommended Exclusive Breast Feeding (EBF) up to six months of age. Before 2001, the WHO recommendation was to continue EBF for four to six months. The WHO definition of EBF is, "feeding an infant only with breast milk, excluding solids or any other fluids (includeing infant formulas) except medicines, vitamins, and minerals" [2]. Up to what age does EBF supports nutriational requirements of a child is debated. Analysis of breast milk samples obtained over 24 hours reveals, $90 \%$ of human breast milk contains water and $100 \mathrm{ml}$ of breast milk contains 70 kilocalories, $1.3 \mathrm{~g}$ of proteins and $50 \mu \mathrm{g}$ of iron [3]. Three to five months after delivery, a lactating mother produces about $750 \mathrm{ml}$ of milk daily which increases to about $800 \mathrm{ml} /$ day after 6 months [4]. Breast milk will provide a child over six months with about 560 calories, $12.7 \mathrm{~g}$ of proteins and $0.4 \mathrm{mg}$ of iron per day. An infant between four to six months needs about $9 \mathrm{~g}$ of proteins, 690 calories and $4.3 \mathrm{mg}$ of iron per day [5]. Accordingly, breast milk provides adequate proteins up to six months, but calories and iron appear to fall short. Although the iron content in breast milk is low, bioavailability is high. Iron in breast milk and iron stores in the baby are often sufficient during the initial months of life [5].

A paper published in British medical journal questioned extending EBF up to six months, suggesting this practice may be harmful than beneficial [6]. WHO promptly responded with counter arguments to support its recommendations. The WHO response was based on a systematic review on optimal duration of EBF, which revealed exclusively breastfeeding adequately supports growth of babies up to six months [7]. Inadequate intake 
of nutrients results in growth faltering, defined as a growth rate below appropriate for a child's age and sex. Growth is interpreted by comparing growth parameters of a child against standard growth charts. Growth charts derived from WHO Multicentre Growth Reference Study (MGRS) are used in Sri Lanka. MGRS was based on the theory "growth of children from birth to five years depends mainly on nutrition, feeding practices, environment and health care than genetics or ethnicity" [8]. If growth charts do not represent the population, growth will be misinterpreted. Mean birth weight for boys according to MGRS is $3.3 \mathrm{~kg}$. According to a study done in Kegalle district in Sri Lanka, mean birth weight was 2.8 $\mathrm{kg}$ [9] and another study done in Gampaha district, it was $2.9 \mathrm{~kg}$ [10]. There are no data for post natal growth of Sri Lankan children to compare with MGRS. Judging by the difference in birth weight between MGRS and Sri Lankan studies, using MGRS data for growth monitoring in Sri Lanka is questionable. EBF rate in Sri Lanka is high compared to other countries in the region [11]. This study was conducted to assess feeding pattern and physical growth during first six months of Sri Lankan babies living in Gampaha district.

\section{MATERIALS \& METHODS}

\subsection{Participant Selection}

A prospective observational study was conducted, where a birth cohort of normal term babies was followed up two monthly, until six months of age. EBF rates among Sri Lankan children between 0 - 5 months was $75.8 \%$ according to Sri Lanka Demographic and Health Survey (DHS) 2006/07 [12]. A sample size of 281 is required to study correlates of EBF to within a margin of sampling error of $5 \%$ (prevalence $70 \%$ and $95 \%$ confidence interval $65 \%-75 \%$ ). Assuming $75 \%$ of the babies invited will complete the study, 400 babies out of normal term babies born in Gampaha district during a period of one month, were randomly selected using computer generated numbers.

\subsection{Data Collection}

Parents of selected babies were contacted over the phone or by post cards, informing about the study. Parents were requested to bring their children to a special clinic conducted at the University Paediatric Unit of North Colombo Teaching Hospital. Participation in the study was voluntary and informed written consent was obtained from mothers at the beginning of the study. Selected babies were followed up at two, four and six months of age. At each visit using a pretested and validated questionnaire administered by a pre-intern doctor, details of feeding since last clinic visit was obtained. Socio-demographic data were obtained at the first visit.
The pre-intern doctor was trained and supervised by the first investigator. Mothers were specifically questioned whether any food, formula milk or water was given to the baby, other than breast milk. A baby given only breast milk for a given period since birth was considered exclusively breastfed up to that age. A baby given any food, drink or water, other than breast milk was considered not exclusively breastfed. Reasons for not practicing EBF were recorded.

Weight and length of all babies were measured by the same pre-intern doctor, assisted by a medical student to avoid inter observer error. Both had one week training under the principle investigator. Weight was measured to the second decimal in kilograms using a beam balance scale standardised once a week. The length was measured to the first decimal in centimetres using a foldable infantometer. Measurements were randomly repeated by the first investigator to ensure accuracy. Kappa scores of one and 0.95 were obtained for weight and length respectively, indicating strong agreement between first investigator and the pre-intern doctor. Growth parameters of babies were compared against growth charts available in the Child Health Development Record (CHDR). Based on MGRS data, these charts have curves representing the median and different Standard Deviations (SD) and areas between median plus 2SD, to the median and minus 1SD is coloured in dark green. The area between minus 1SD to minus 2SD is light green. The area between minus $2 \mathrm{SD}$ to minus $3 \mathrm{SD}$ is orange, while area below minus 3SD is red. Area above plus 2SD is purple. According to a general circular issued by Ministry of Healthcare and Nutrition in Sri Lanka [13], "inadequate weight gain, no weight gain or drop in weight, between two successive weight measurements" is defined as growth faltering. Nothing mentioned about length. Terms "no weight gain and drop in weight" are clear but "inadequate weight gain" is vague. Generally a child dropping from one colour zone to a lower zone is considered as growth faltering. Accordingly a child just above a SD line, dropping just below it will also be considered having growth faltering. In this study, we used an objective method to define growth faltering. At each age one third of the width of each colour zone was measured. A growth parameter lying above one third of the width of a colour zone, dropping lower than the upper one third of lower colour zone during next measurement was considered as growth faltering. Catch up growth was defined as a child with growth faltering reaching a higher colour zone on subsequent measurements.

All babies were also examined by a consultant Paediatrician or a senior registrar at each visit. In children with growth faltering appropriate actions were taken when there was an identifiable cause. When EBF was stopped without an indication mothers we reencouraged to restart 
EBF. All babies were invited for the next follow up irrespective of their growth or feeding pattern. Decision to stop or continue EBF was taken by mothers, but EBF was promoted at all times. Longitudinal analysis of data was done. Growth faltering pattern and association between feeding pattern on growth were assessed. The StaTistical Package for Social Sciences (SSPS) version 16 was used for data analysis.

\subsection{Ethical Issues}

Ethical clearance was obtained from the ethics committee of Faculty of Medicine, University of Kelaniya. Informed written consent was obtained from mothers. Appropriate action was taken for babies with medical or surgical problems. Correct feeding advice was given to mothers and EBF was promoted at all times. Apart from travelling expenses, there were no additional expenses to parents.

\section{RESULTS}

Although 400 babies selected only 372 were presented at two months. At four and six months 330 and 286 babies were presented respectively. Babies were from a socio-economically mixed background. Socio-demographic characteristics of babies who completed all three follow up visits are summarized in Table 1. Socio-demographic characteristics of the babies who dropped out were also assessed and compared with the babies who completed the follow up. There was no significant difference between two groups.

Of the babies presented at two months, 366 (98.3\%) were on EBF and 41 (11.2\%) of them had weight faltering. Of these babies 12 had problems establishing breastfeeding initially, but did not have further GF with correction of feeding technique. Three babies with GF during this period had respiratory tract infections. Majority (63.4\%) with GF during this period, had no identifiable cause. Four babies had formula feeding in addition to breast milk due to weight faltering and all had a catch up growth. Four children were started on formula milk for other reasons and none had weight faltering. Weight and length faltering observed at different ages is summarized in Table 2.

Of the babies presented at four months, 249 (75.5\%) were on EBF. Out of 41 babies who had weight faltering while on EBF at two months, 32 were presented at four months. Of them 23 were still on EBF. Seven of them had a catch up growth, while others had no further weight faltering. Of the babies on EBF up to four months, $50(20.1 \%)$ had weight faltering between two and four months. Out of them, eight babies had infections in the respiratory or urinary tract during this period, while no cause was found in the rest $(84 \%)$. Between two and four
Table 1. Socio-demographic characteristics of babies followed up till six months.

\begin{tabular}{|c|c|}
\hline Characteristic & Frequency \\
\hline \multicolumn{2}{|c|}{ Mother's employment status } \\
\hline Formally employed & $49(17.3 \%)$ \\
\hline Not formally employed & $237(82.7 \%)$ \\
\hline \multicolumn{2}{|c|}{ Mother's age } \\
\hline$<30$ years & $152(53.1 \%)$ \\
\hline$>30$ years & $134(47.9 \%)$ \\
\hline \multicolumn{2}{|c|}{ Father's education } \\
\hline Up to Grade 5 & $07(2.5 \%)$ \\
\hline Grade 6 to 11 & $191(67.1 \%)$ \\
\hline Grade 12 to 14 & $79(27.3 \%)$ \\
\hline University & $09(3.1 \%)$ \\
\hline \multicolumn{2}{|c|}{ Mother's education } \\
\hline None & $01(0.4 \%)$ \\
\hline Grade 1 to 5 & $06(2.1 \%)$ \\
\hline Grade 6 to 11 & $180(62.9 \%)$ \\
\hline Grade 12 to 14 & $90(31.5 \%)$ \\
\hline University & $09(3.1 \%)$ \\
\hline \multicolumn{2}{|c|}{ Monthly family income( SLR) } \\
\hline$<9999$ & $19(6.6 \%)$ \\
\hline $10,000-19,999$ & $104(36.4 \%)$ \\
\hline $20,000-34,999$ & $97(33.9 \%)$ \\
\hline$>35,000$ & $66(23.1 \%)$ \\
\hline
\end{tabular}

months 43 babies stopped EBF due to growth faltering, but only $25(58.1 \%)$ of them had a catch up growth. EBF was stopped in 32 babies for other reasons and three out of them had weight faltering.

Of the babies presented at six months 204 were on EBF. Of them, 27 (13.2\%) had weight faltering between four and six months. Of the babies who had growth faltering between two and four months while on EBF, 31 were presented at six months. Of these babies, 24 were still on EBF and four of them had a catch up growth. Only three babies had further growth faltering between four and six months. EBF was stopped in 25 babies during four and six months. Of these, eight babies stopped EBF because of weight faltering but only three of them $(37.5 \%)$ had a catch up growth. No weight faltering was observed in 17 babies who stopped EBF for other reasons. Effect on weight faltering by stopping EBF is shown in Table 3. Of the 27 babies with weight faltering while on EBF, two had viral infections, two had bronchiolitis, one had a febrile convulsion and one had gastroenteritis during this period, but no cause was identified in $77.7 \%$.

At two months 57 (15.6\%) babies on EBF had length faltering compared to birth. Of these babies, 45 were 
Table 2. Growth faltering observed among exclusively breastfed babies at different ages.

\begin{tabular}{cccc}
\hline Age & Number exclusively breast fed & Number with weight faltering & Number with length faltering \\
\hline 0 - 2 months & 372 & $41(11.2 \%)$ & $57(15.6 \%)$ \\
2 - 4 months & 249 & $50(20.1 \%)$ & $55(22.1 \%)$ \\
$4-6$ months & 204 & $27(13.2 \%)$ & $34(16.5 \%)$ \\
\hline
\end{tabular}

Table 3. Effect on growth faltering by stopping EBF (EBF).

\begin{tabular}{cccc}
\hline Age period & $\begin{array}{c}\text { Number presented at } \\
\text { each follow up }\end{array}$ & $\begin{array}{c}\text { Number stopped EBF due to } \\
\text { weight faltering }\end{array}$ & $\begin{array}{c}\text { Number had catch up growth } \\
\text { by stopping EBF }\end{array}$ \\
\hline 0 to 2 months & 372 & 04 & $04(100 \%)$ \\
2 to 4 months & 330 & 43 & $25(58.1 \%)$ \\
4 to 6 months & 286 & 08 & $03(37.5 \%)$ \\
\hline
\end{tabular}

presented at four months and 33 were still on EBF and eight of them had a catch up growth in length. None had further length faltering. Out of exclusively breastfed babies at four months, $55(22.1 \%)$ had length faltering between two and four months. Of these 55 babies, 26 were presented at six months and 23 of them were still on EBF. Of them five showed a catch up growth and three had further length faltering. Others remained in their new colour zone. During four and six months $16.5 \%$ of exclusively breastfed babies had length faltering. None had length faltering recorded at all three clinic visits. At all ages length faltering rate was higher than the rate of weight faltering. All babies who had length faltering had weight faltering as well. Addition to our special clinics children attended routine child welfare clinics (CWCs) conducted by medical officer of health in their area and growth was recorded there as well. Decision of mothers to stop EBF due to growth faltering was based on weight measured at these clinics. Our weight measurements and child welfare clinic weight measurements were comparable. Length was not recorded at most of CWCs. When recorded, length measurements at child welfare clinics were wrong most of the time and always higher than our recordings. We found that the discrepancy was due to not using an infantometer at CWCs.

\section{DISCUSSION}

In contrary to concerns expressed by Fewtrell et al. [6], EBF seems to support growth in the majority of babies up to six months. Fewtrell et al. were of the view that extending EBF up to six months will result in significant growth faltering between four and six months and iron deficiency anaemia. In our study, highest rate of growth faltering among exclusively breastfed babies was noted between two and four months. Only a small percentage of EBF babies had growth faltering between four and six months.
Although breast milk contains low iron compared to formula milk, absorption from breast milk is two to three times higher. When the mother is iron deficient during pregnancy and lactation, iron deficiency in infant is possible. We are unable to comment about iron status of exclusively breastfed babies because we have not addressed that issue in this study. Iron deficiency among exclusively breastfed babies can be prevented by iron supplementation of at risk babies, rather than stopping EBF [5].

All babies who stopped EBF between birth and two months due to growth faltering showed a catch up growth, but this was not the case in other two age categories. This indicates growth faltering can be due to reasons other than inadequate nutrition. Few babies with growth faltering had various infections, but in the majority no identifiable reason was found. A large percentage of babies who showed growth faltering at some stage, but continued EBF did not show further growth faltering.

Presently MGRS growth charts are used for growth monitoring in Sri Lanka. In a study conducted by the same authors to assess growth parameters at birth of normal term babies in Sri Lanka revealed, means of all three growth parameters were falling near mean minus one standard deviation on MGRS curves [14]. Abesena et al. [10] also showed a similar trend. The difference between MGRS data and data from Perera et al. can only be explained by possible genetic influence as in both studies babies with maximal growth potentials were included. A twin study from Gambia has also shown that genetics do play a role in addition to nutrition in regulation of physical growth [15]. Further studies are needed to prove this fact decisively. If growth faltering is being over diagnosed, a significant number of babies loose advantages of EBF unnecessarily. Obesity will result in trying to push up growth of otherwise normal children, who are destined to be small genetically by providing extra food. 
On the other hand growth faltering corrected with adding formula milk does not necessarily indicate those babies having inadequate breast milk. Unlike with breast feeding, overfeeding can occur with formula milk, resulting in providing excess nutrition. This can place a child higher up in the weight charts than his genetic potential. During immediate period after birth mother produce colostrum which is produced in small quantities, but adequate for the baby. Due to maternal anxiety and poor attachment, there can be feeding failure resulting in growth faltering. This is often not due to inadequate breast milk produced by the mother, but due to other factors associated with breastfeeding. Starting formula milk will naturally induce a catch up growth in these babies, but if feeding techniques were corrected, some of these babies would show a catch up growth even without formula milk.

There are some instances where EBF is not sufficient to support nutrition of a baby. Growth faltering alone is not adequate to identify these babies. Stopping EBF in a child with growth faltering should be only after considering all factors influencing physical growth. Especially genetic factors should be considered by measuring the height of parents. This will minimize stopping of EBF unnecessarily and the possible risk of obesity. Unfortunately majority of mothers stop EBF on their own once growth starts to falter [11]. If growth charts relevant for local population are available for growth monitoring, unnecessary stopping of EBF can be minimized.

Stunting during childhood would lead to reduced final adult height. Although length charts are available in the CHDR, length is not recorded routinely. A plastic tape instead of infantometer is used for length measurements at CWCs. This explains the higher lengths recorded at CWCs compared to our readings. Use of infantometer at CWCs should be made compulsory. Measuring error in length leads to underestimation of stunting and over diagnosing of wasting. Though not happening at present, length should be considered when nutritional interventions are planned. This is important because at all ages the rate of length faltering was more than weight faltering.

Comparison of babies on EBF and not on EBF was not done, because majority of the babies not on EBF were on EBF at some time and one reason to stop EBF was growth faltering. It is unethical not to give EBF to a randomly selected group of children for research purpose. In a country like Sri Lanka where EBF is high, it is impossible to find an adequate sample of babies not on EBF from birth to compare with exclusively breast fed babies.

Extension of EBF up to six months reduces the incidence of diarrhoeal diseases, which causes significantmorbidity and mortality in developing countries [7]. As majority of the babies maintained adequate growth up to six months with EBF, it is advisable to continue EBF up to six months with accurate and careful growth monitoring.

\section{LIMITATIONS}

Main limitation in our study was the dropouts in between follow ups. All attempts were made to get babies for follow up. The socio-demographic data of babies who dropped out and babies who completed six months follow up had no significant difference. Longitudinal analysis was done to minimize the error due to dropouts.

\section{REFERENCES}

[1] Lucas, A. (1998) Programming by early nutrition: An experimental approach. Journal of Nutrition, 128, 401406S.

[2] World Health Organization (WHO) (2012) Infant and young child nutrition. 55th World Health Assembly, 2002. http://apps.who.int/gb/archive/pdf_files/WHA55/ewha55 25.pdf

[3] McIntosh, N., Helms, P. and Smyth, R. (2003) Forfar \& Arneil's text book of paediatrics. 6th Edition, Elsevier, London, 1909-1910.

[4] Breastfeeding.about.com. (2013) The contents and composition of breast milk.

breastfeeding.about.com/od/breastfeedingbasics/p/bmcontent. $\mathrm{htm}$

[5] Kliegman, R.M., Jenson, H.B., Behrman, R.E. and Stanton, B.F. (2007) Nelsons 271 text book of pediatrics. Saunders, Philadelphia, 210-213.

[6] Mary Fewtrell, M. and Wilson, D.C. (2011) Booth I. Six months of EBF: How good is the evidence? BMJ, 342, Article ID: c5955. doi:10.1136/bmj.c5955

[7] Kramer, M.S. and Kakuma, R. (2004) The optimal duration of EBF: A systematic review. Advances in Experimental Medicine and Biology, 554, 63-77. doi:10.1007/978-1-4757-4242-8_7

[8] de Onis, M., Onayongo, A., Broeck, J., Chumlee, C. and Matorell, R. (2004) Measurement and standardization protocols for anthropometry used in the construction of a new 2 international growth reference. Food and Nutrition Bulletin, 25, S15-S26.

[9] World Health Organization (1984) Perinatal mortality \& morbidity including low birth weight. South East Asian Regional Organization, New Delhi.

[10] Abeysena, C., Payawardana, P. and Senevitathne, R. (2009) Maternal sleep deprivation is a risk factor for small for gestational age: A cohort study. Australian and New Zeeland Journal of Obstetrics and Gynecology, 49, 382-387.

[11] Perera, P.J., Fernando, M., Warnakulasuria, T. and Ranathunga, N. (2011) Feeding practices amongchildren attending child welfare clinics in Ragama $\mathrm{MOH}$ area: A descriptive cross-sectional study. International Breastfeeding Journal, 6, 18.

www.internationalbreastfeedingjournal.com/content 
doi:10.1186/1746-4358-6-18

[12] Department of Census and Statistics in Collaboration with Ministry of Healthcare and Nutrition (2008) Sri Lanka demographic and health survey 2006/7. Department of Census and Statistics.

[13] Ministry of Healthcare and Nutrition (2008) Protocol for managing nutritional problems among under five children in the community. General circular letter no 02-18/2008.

[14] Perera, P.J., Fernando, M., Warnakulasuria, T. and Rana- thunga, N. (2012) Statistical characteristics of growth parameters at birth of babies born in Gampaha district in Sri Lanka. Oral Presentation at Asia-Pacific Public Health Consortium, Colombo.

[15] Jepson, A., Banga, W., Hassan-King, M., Sisay, F. and Bennets-Whittle, H. (1994) Twin children in Gambia: Evidence for genetic regulation of physical characteristics in the presence of sub-optimal nutrition. Annals of Tropical Paediatrics, 14, 309-313. 